\title{
Tecnologia e degradação de pastagens na pecuária no Cerrado brasileiro
}

\section{Technology and degradation of pastures in livestock in the brazilian Cerrado}

\author{
Elis Regina de Oliveira \\ Janete Rego Silva ${ }^{2}$ \\ Luis Rodrigo Fernandes Baumann ${ }^{3}$ \\ Fausto Miziara 4 \\ Laerte Guimarães Ferreira 5 \\ Leonardo Rodrigues de Oliveira Merelles ${ }^{6}$
}

Palavras-chave:

Índice tecnológico

Indice degradação pastagens

Pecuária

Análise correspondência múltipla

Bacia do Rio Vermelho

\begin{abstract}
Resumo
A pecuária no Brasil teve papel relevante na formação do espaço rural. Tal processo ocasionou um passivo ambiental, pois se desenvolveu sem considerar os impactos ao meio ambiente, relativos à erosão e compactação dos solos, emissão de gases de efeito estufa (GEE), além do uso intensivo de terra e dos recursos hídricos. Em significativa parcela das propriedades o manejo inadequado resultou em pastagens com níveis de degradação. A literatura aponta a importância da intensificação da tecnologia como forma de garantir pastagens sustentáveis. Esta pesquisa tem por objetivo analisar a relação entre nível tecnológico de produção de bovinos e de degradação de pastagens na Bacia Hidrográfica do Rio Vermelho, em Goiás - Brasil. Esta área é considerada como representativa da pecuária no cerrado brasileiro. Foi utilizada amostra estratificada por compartimentos com 60 estabelecimentos agropecuários. Utilizaram-se as técnicas de análise de correspondência múltipla, análise de cluster e regressão beta para obter o Índice de Degradação de Pastagens (IDP). Verificaram-se associações bivariadas entre o Índice Tecnológico (IT) e as variáveis: renda, relevo e tamanho da propriedade. Foi possível constatar o emprego de maior nível tecnológico nas maiores propriedades e em terrenos mais planos. Também se constatou que o maior nível tecnológico propicia maior remuneração aos gestores. Porém não se observou relação entre a tecnologia empregada nos estabelecimentos e taxa de lotação animal, o que pode comprometer os resultados econômicos e bem-estar animal. Assim, como não foi observada associação entre índice tecnológico e índice de degradação de pastagens, sugerindo ocorrência de
\end{abstract}

${ }^{1}$ Universidade Federal de Goiás, Goiás, Brasil. elisregina@pucgoias.edu.br

2 Universidade Federal de Goiás, Goiás, Brasil. janetegt25@gmail.com

Universidade Federal de Goiás, Goiás, Brasil. fernandesbaumann@gmail.com

${ }^{4}$ Universidade Federal de Goiás, Goiás, Brasil. faustomiziara@uol.com.br

${ }^{5}$ Universidade Federal de Goiás, Goiás, Brasil. lapig.ufg@gmail.com

${ }^{6}$ Pontifícia Universidade Católica de Goiás, Goiás, Brasil. leonardotringo@gmail.com 
degradação de pastagens, mesmo quando do uso de tecnologias na produção de bovinos.

\section{Keywords:}

Technological index

Pasture degradation index

Cattle ranching

Multiple Correspondence Analysis

Vermelho River basin

\begin{abstract}
In Brasil ranching has played a fundamental role in the occupation of rural land. This process results in a series of environmental impacts, such as the erosion and compaction of the soil, and the emission of greenhouse gases, in addition to the intensive use of the soil and water resources. In part of the properties, inadequate management resulted in pastures with degradation levels. The literature points out the importance of intensifying technology as a way to guarantee sustainable pastures. The present study aims to identify and analyze the relationships between the technological level level of cattle production and the degradation of pastures in the Vermelho River basin in Goiás, central Brazil. This area is considered to be representative of livestock in the Brazilian Cerrado. The sample consisted of 60 cattle ranches, selected from each of the three sectors of the Vermelho River basin. The data were analyzed using a Multiple Correspondence Analysis, cluster analysis, and Beta regression to obtain Pasture Degradation Indice (PDI). Bivariate associations were found between the Technological Index (TI) and three variables - the manager's income, topographic relief, and the size of the property. It was possible to verify the use of a higher technological level in the largest properties as well as in flatter areas. It was also found that the higher technological level provides higher remuneration to managers. However, there was no relationship between the technology used in the establishments and the cattle density, which can reduces profitability economic and animal welfare. There was also no association between TI and PDI, suggesting pasture degradation, even when using more intensive technologies in cattle production.
\end{abstract}

\section{INTRODUÇÃO}

A pecuária nos países em fase de desenvolvimento teve papel relevante na definição de estratégias institucionais de ocupação do espaço rural e no avanço da fronteira agropecuária. Em geral, constituiu-se no primeiro uso econômico do solo, após exploração da madeira, resultando em conversão da vegetação nativa em pastagens, para então ceder espaço para atividades agrícolas e formação de cidades (GIBBS et al., 2010; GOENER et al.,2007; GRIFFITH, 2016; PINHEIRO et al., 2016).

Tal processo ocasionou passivo ambiental, pois se desenvolveu sem considerar os impactos ao meio ambiente, relativos a erosão e compactação dos solos, emissão de gases de efeito estufa (GEE), além do uso intensivo de terra e dos recursos hídricos. O manejo inadequado das pastagens e ausência de técnicas de conservação resultou em degradação (ARMENTERAS et al., 2013; DIAS et al., 2016)..

A pecuária bovina brasileira pode ser direcionada pela finalidade de geração de renda, reserva de valor imobiliário (especulação) ou lazer (MARGULIS, 2003). As diferentes características de clima, topografia, solos, fase de desenvolvimento do animal (criarecria-engorda) e condições socioeconômicas exigem distintas soluções tecnológicas, para a intensificação da produção de gado de corte no país (DIAS et al., 2016; MCMANUS et al., 2016). As estratégias tecnológicas voltadas para intensificação podem estar relacionadas com melhoramento genético, nutrição, saúde animal e manejos.

O processo de intensificação da produção de gado de corte está pressionado pelo esgotamento do avanço da fronteira 
agropecuária, por maior controle ambiental, pelo crescimento da demanda por terras agricultáveis e por proteína animal (ARMENTERAS, 2013; OECD-FAO, 2015). Assim, a intensificação conduz ao uso mais racional dos recursos naturais, podendo ser poupadora das áreas com vegetação nativa remanescente, inclusive floresta e redutora da emissão de gases de efeito estufa, em virtude da redução do ciclo produtivo, além de acelerar o fluxo de receitas (CARO et al., 2017).

A tomada de decisão do gestor por intensificar a produção de bovinos pode ser influenciada pelo seu perfil socioeconômico, a finalidade da atividade, fase de desenvolvimento do animal; preço da arroba do boi, coordenação entre os agentes da cadeia produtiva da carne e disponibilidade dos fatores de produção (BOWMAN et al., 2012). O gestor da propriedade precisa compreender as interações entre relevo-solo-clima-plantaanimal-mercado. Portanto, essa atividade exige do gestor uma abordagem sistêmica e multidisciplinar.

Essa pesquisa tem por objetivo identificar e analisar a relação entre nível tecnológico e nível de degradação de pastagens com alguns dos fatores que condicionam a tomada de decisão do produtor de bovino, utilizando como unidade geográfica a Bacia Hidrográfica do Rio Vermelho (BHRV). Em conformidade com a fundamentação teórica e metodologia, são analisadas respostas para as seguintes perguntas:

a) $\mathrm{O}$ nível tecnológico adotado pela unidade produtora de bovinos na BHRV depende do nível de educação e de renda do seu gestor? Depende da disponibilização de crédito rural?

b) O relevo percebido pelo gestor da BHRV está correlacionado com o nível tecnológico adotado para o sistema de produção de bovino?

c) Propriedade com sistema produtivo de bovino com maior nível tecnológico apresenta menor degradação de pastagens?

d) O tamanho da propriedade está correlacionado com o nível tecnológico adotado pelos gestores?

e) O tamanho da propriedade está correlacionado com o nível de degradação de pastagens?

f) As maiores taxas de lotação ocorrem em sistemas com maior nível tecnológico?
A bacia hidrográfica do Rio Vermelho (BHRV), situada no Oeste do estado de Goiás, região central do Brasil, com área de aproximadamente $11 \mathrm{mil} \mathrm{km}^{2}$, está totalmente inserida no bioma Cerrado. O uso do solo da BHRV evidencia o predomínio de pastagem, destinada principalmente à pecuária bovina, com áreas esparsas destinadas à lavoura, com concentração de pivô em algumas delas, indicando lavoura irrigada.

Predomina, também, a produção de bovinos da raça nelore criados em sistema de pastejo, baseado no cultivo de capim Brachiária, que são adaptados às condições tropicais, em solos de baixa e média fertilidade (KLINK; MACHADO, 2005; ROSA, et al., 2001).

\section{MATERIAL E MÉTODOS}

A coleta de dados foi realizada por meio de formulário, estruturado em conformidade com as hipóteses dessa pesquisa, considerando três dimensões básicas: 1) Perfil socioeconômico do gestor do estabelecimento agropecuário; 2) caracterização do sistema produtivo; e 3) caracterização de manejo de pasto e condições ambientais.

A bacia hidrográfica do Rio Vermelho (BHRV) foi compartimentalizada em três seções, considerando declividade e o perfil topográfico. A declividade ao longo da bacia varia fortemente no sentido sul-norte com valores mais acentuados, expressando relevo mais movimentado, com suavização ao longo da bacia na direção norte (Figura 1).

Os compartimentos estão caracterizados em Alta bacia - com altitudes acima de 500m, com declividades mais acentuadas (45 a 20\%), maior diversificação de solos e alta vulnerabilidade ambiental; Média bacia, com altitude altitudes variando de 350 a $500 \mathrm{~m}$ e com declividade de 8 a 20\%; Baixa bacia - com altitude abaixo de $350 \mathrm{~m}$, declividade abaixo de 8\% (MACHADO; LIMA, 2011).

E em seguida os pontos foram distribuídos, considerando a maior variabilidade de declividade e de solos, com a finalidade de melhor representação das características biofísicas dos sistemas produtivos de bovinos, resultando em 25 pontos de coleta para a Alta bacia, 24 para a Média bacia e 11 para a Baixa bacia. 
Figura 1 - Mapa de compartimentos de relevo e dos pontos de coleta do questionário, da BHRV GO, 2016.

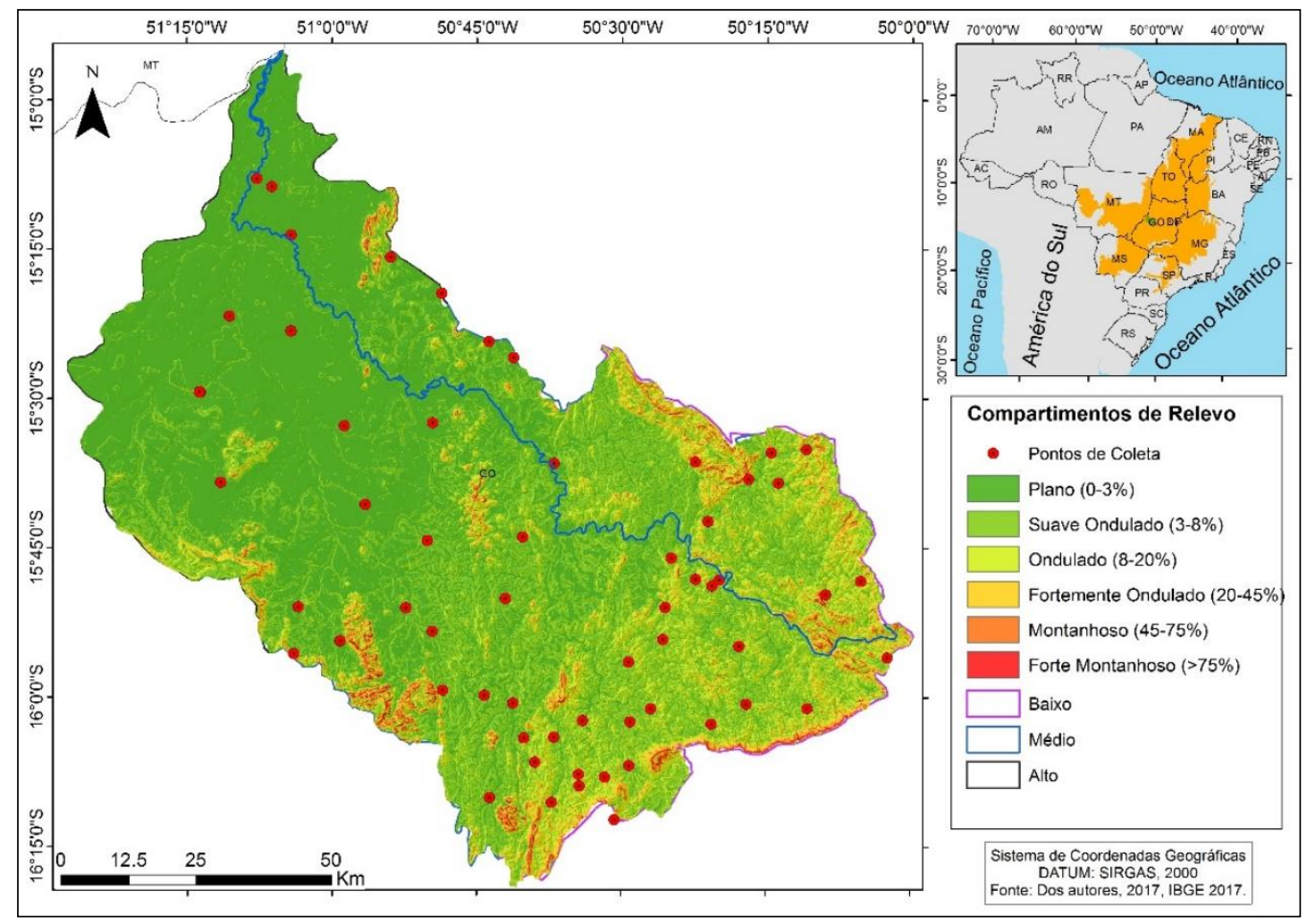

Fonte: IBGE (2017). Org.: pelos autores, 2017.

\section{Tratamento e Análise dos Dados}

Os índices de nível tecnológico e de degradação de pastagens foram construídos, para então avaliar a associação entre eles e as demais variáveis, conforme exposto nesta seção. A construção do Índice Tecnológico (IT) está detalhada em Oliveira et al. (2018).
Conforme Tabela 1, para a construção do Índice de Degradação de Pastagens (IDP), foram selecionadas cinco variáveis indicadoras de redução de produtividade e de degradação de pasto, conforme literatura, com total de 12 categorias (LIMA et al., 2012; PERON; EVANGELISTA, 2004).

Tabela 1 - Variáveis utilizadas para geração do IDP

\begin{tabular}{|c|c|}
\hline Variável & Classes \\
\hline solos expostos (se) & (não: $0 ; \operatorname{sim}: 1$ ) \\
\hline processos erosivos (pe) & (não: $0 ; \operatorname{sim}: 1$ ) \\
\hline $\begin{array}{l}\text { presença de cupim de } \\
\text { montículos }(p c)\end{array}$ & (não: $0 ; \operatorname{sim}: 1$ ) \\
\hline altura do capim (ac) & (abaixo de $12 \mathrm{~cm}$ : 0; acima de $12 \mathrm{~cm}: 1$ ) \\
\hline $\begin{array}{l}\text { presença de plantas } \\
\text { invasoras (pi) }\end{array}$ & $\begin{array}{l}\text { pi:0 não existe; pi: } 1 \text { existe em até } 15 \% \text { da } \\
\text { área; pi: } 2 \text { acima de } 15 \% \text { ) }\end{array}$ \\
\hline
\end{tabular}

Org:. pelos autores, 2017.

Para a construção desses índices aplicou-se a técnica de Análise de Correspondência Múltipla (ACM) para obtenção das componentes com maior proporção da variabilidade explicada, gerando um índice que represente o nível de degradação de pastagens dos estabelecimentos agropecuários (GUEDES et al., 2008; HAIR JR. et al., 2009; PAGÈS, 2014). Utilizou-se o software $R$ para realização da ACM, com a matriz de Burt ajustada, assim como os demais tratamentos estatísticos (GREENACRE, 2007). 
Em seguida aplicou-se análise de cluster hierárquico com método de Ward, gerando os agrupamentos das variáveis e dos estabelecimentos agropecuários (fazendas) (HAIR JR. et al., 2009; WARD JR., 1963). Aplicou-se, também, regressão Beta com o objetivo de replicar o IDP para outras fazendas da região (OLIVEIRA et al., 2018).

Utilizou-se, também, o Índice Moran como técnica de verificação de dependência espacial, com o objetivo de avaliar a existência de autocorrelação no IDP, referente à região geográfica estudada (LIMA et al., 2014; OLIVEIRA et al., 2018; SHIRVANI et al, 2017).

Para analisar e testar a dependência entre as variáveis foi aplicado os testes nãoparamétricos de medida de correlação de Spearman $\left(\rho_{s}\right)$, e posto de Kendall tau_b $\left(\tau_{b}\right)$ e tau_c $\left(\tau_{c}\right)$, considerando $\mathrm{H}_{0}:\left(\rho_{\mathrm{s}}\right.$ ou $\tau_{\mathrm{b}}$ ou $\left.\tau_{\mathrm{c}}\right)=0 \mathrm{e}$ a hipótese alternativa bilateral com $\mathrm{H}_{1}$ : $\left(\rho_{\mathrm{s}}\right.$ ou $\tau_{\mathrm{b}}$ ou $\left.\tau_{\mathrm{c}}\right) \neq 0$; e o teste exato de dependência de Fisher.

\section{RESULTADOS E DISCUSSÃO}

O IT foi obtido utilizando-se a análise de Correspondência Múltipla (ACM), Análise de Cluster e Regressão Beta. A construção deste índice está detalhada em Oliveira et al. (2018).

A ACM conjugada com a Análise de Cluster, considerando o conjunto de variáveis indicadoras de nível tecnológico, revelaram duas estratégias distintas de investimento em nutrição: a) com investimento em formação e manutenção de pastagens, considerando uso de fertilizante, análise de solo, uso de calcário, análise bromatológica do capim, e uso de trator com potência acima de $100 \mathrm{cv}$; e b) com investimentos em suplementação, principalmente no período de seca, para compensar a redução de oferta e baixo valor nutricional das pastagens, uso de assistência técnica, trator até $100 \mathrm{cv}$, entre outras. Nesse agrupamento predominam variáveis de suplementação, conjugada com pastejo, com taxas de lotação de até duas (UA/hectare). A maior taxa de lotação (acima de 2 UA/ha) está agrupada com as demais variáveis indicadoras de baixo nível tecnológico.

Foram observados três agrupamentos para as variáveis e para os estabelecimentos agropecuários, com variáveis indicadoras de baixo, médio e alto nível tecnológico. Verificou- se que na Baixa e na Média bacia predominam os índices de médio e alto nível de tecnificação. Ressalta-se que nelas ocorre, também, predomínio de propriedades com maior área (IBGE, 2006), com maior proporção de relevo e solos mais favoráveis à produção de bovinos (OLIVEIRA et al., 2018).

\section{Índice de degradação de pastagem (IDP)}

Considerou-se as variáveis indicadoras de degradação de pastagens (Tabela 1) para desenvolver a ACM, obtendo duas componentes com explicação da inércia total de $62,7 \%$, para o conjunto de variáveis. A componente principal (1) explicou $52,9 \%$ da inércia total, enquanto a segunda componente explicou 9,8\% (Figura 2). Os coeficientes percentuais de contribuições absolutas indicaram que as variáveis mais relevantes para formação da primeira componente foram solo exposto (se:1) e processo erosivo (pe:1), com contribuições absolutas de $32,54 \%$ e $30,45 \%$, respectivamente.

Verificou-se que a primeira componente expressa possível perda de solo, portanto denominada dimensão representativa de degradação de solo. Enquanto que a segunda componente expressa as condições de degradação biológica do pasto, sendo as variáveis mais relevantes para a sua composição: presença de planta invasora acima de $15 \%$ (pi:2) com $27,73 \%$, seguida por ausência de planta invasora (pi:0) com $27,75 \%$ e altura do capim abaixo de $12 \mathrm{~cm}$ (ac:0), com 25,35\% do total da inércia desse eixo.

Com o auxílio da análise de cluster percebeu-se que a utilização de apenas uma dimensão para modelar o Índice de Degradação da Pastagem (IDP) geraria perda significante de informações, então decidiu-se por trabalhar com os dois eixos principais: o Índice de Degradação de Solo (IDS) (componente 1) e o Índice de Degradação Biológica (IDB) (componente 2), para juntos representar o IDP.

Conforme Figura 3 foram obtidos quatro grupos de variáveis. O primeiro grupo composto pelas variáveis que indicam degradação do solo, pelas presenças de processo erosivo e solos expostos, portanto pior nível de degradação. $\mathrm{O}$ segundo grupo, composto pelas variáveis que indicam melhor manejo da pastagem, pelas ausências de cupim e de plantas invasoras, o que sugere menor nível degradação biológica. 
Figura 2 - ACM com matriz de Burt ajustada para as variáveis indicadoras de degradação de pastagens dos sistemas produtivos de bovinos, BHRV GO, 2016.

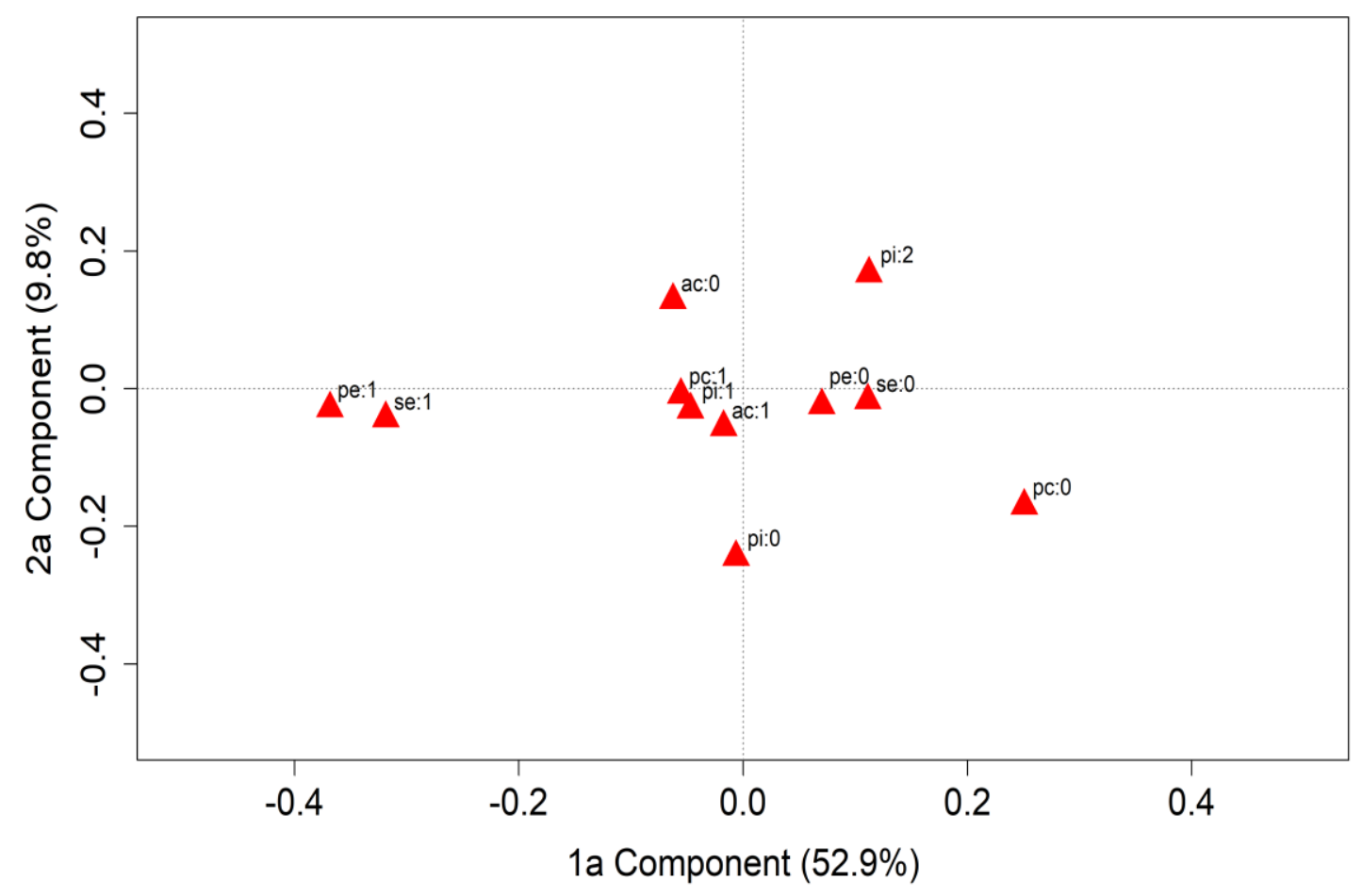

Org.: pelos autores, 2017.

Figura 3 - Agrupamento das variáveis indicadoras de degradação das pastagens, conforme componentes 1 e 2 obtidas pela ACM, para sistemas de produção de bovinos, BHRV GO, 2016.

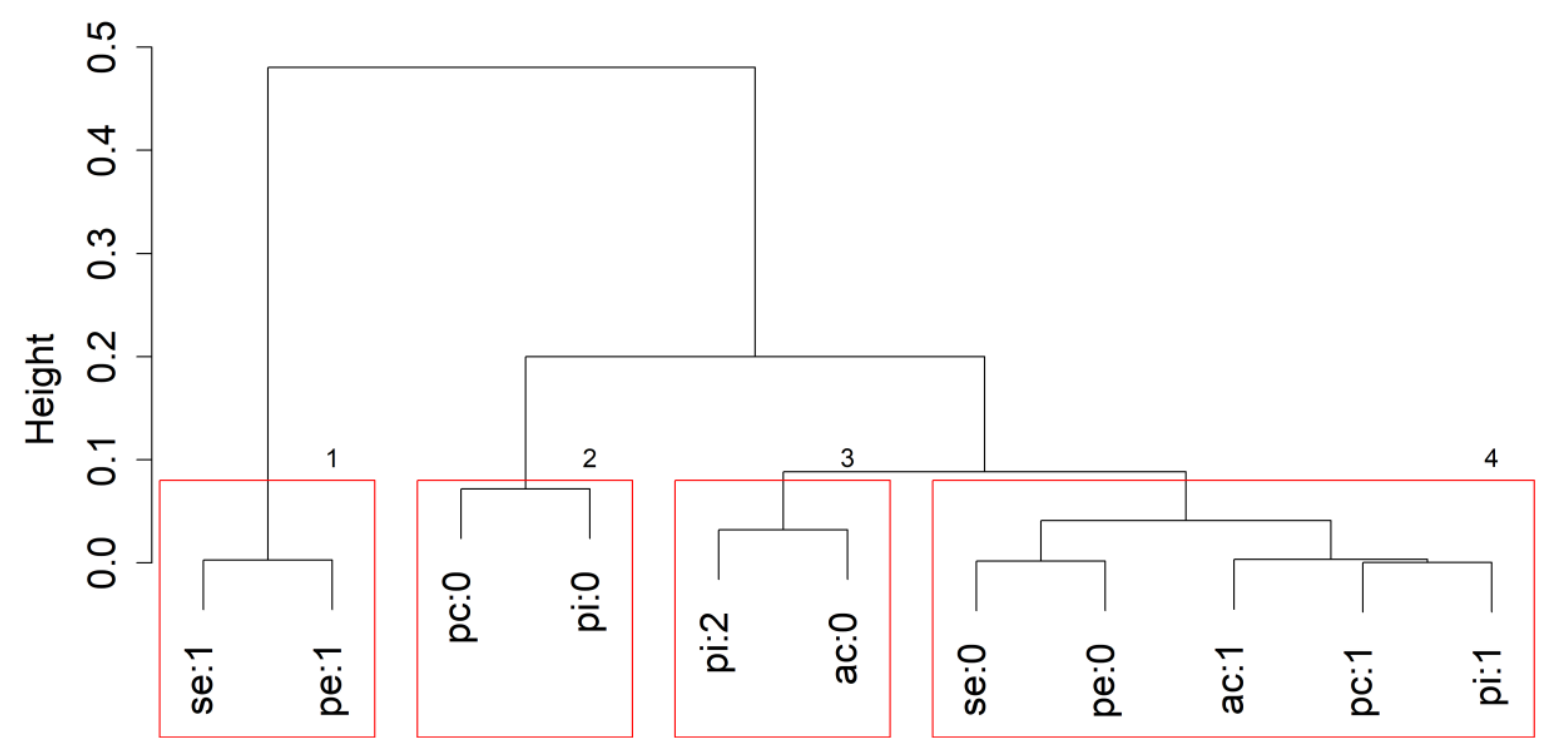

Org.: pelos autores, 2017.

O terceiro grupo representa o conjunto de variáveis com as condições mais inadequadas de manejo das pastagens pela maior presença de plantas invasoras, acima de $15 \%$ da área de pastagem, e altura do capim abaixo de $12 \mathrm{~cm}$, podendo ser classificado como o grupo de variáveis que representam o pior nível de degradação biológica, dessa região. Quarto grupo composto pelas variáveis que sugerem condições regulares de manejo de pastagens, pois é composto pela presença de cupim, de invasora em até $15 \%$ da área de pastagem, com altura do capim acima de $12 \mathrm{~cm}$ e ausência de processo erosivo e de solo exposto. Portanto, o quarto grupo, com maior conjunto de variáveis 
foi classificado como nível regular de degradação biológica.

A análise de cluster relativa às unidades amostrais (estabelecimentos agropecuários) gerou quatro agrupamentos com características semelhantes dentro de cada grupo e distintas entre si, conforme Figura 4, em função do agrupamento das variáveis.

Figura 4 - Agrupamento das fazendas, em função do IDP, conforme coordenadas principais das componentes 1 e 2 das fazendas, para sistemas de produção de bovinos, BHRV GO, 2016.

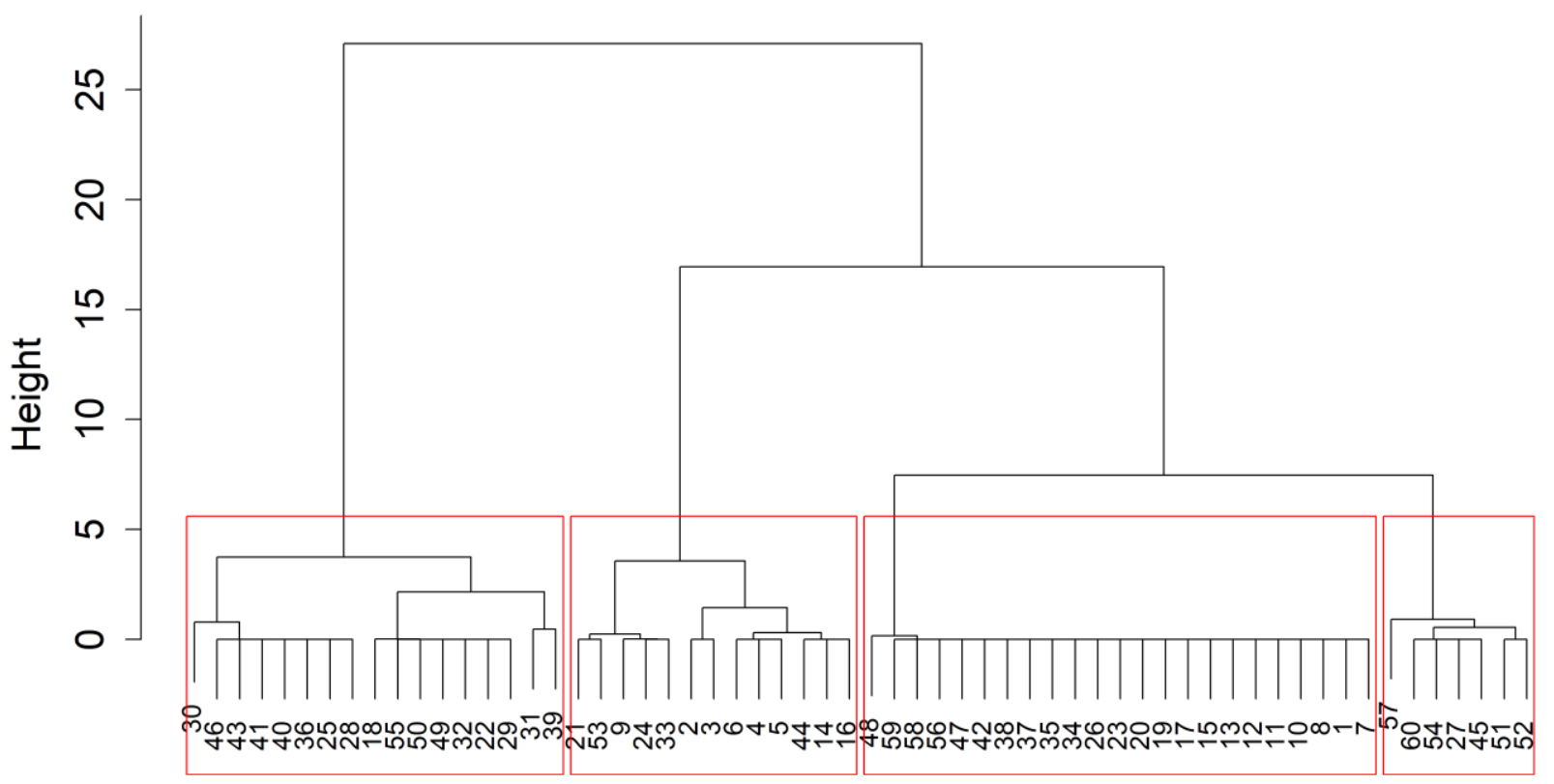

Org.: pelos autores, 2017.

No primeiro grupo, das 17 fazendas $94,12 \%$ das propriedades apresentam solo exposto, 58,82\% com presença de processo erosivo, $100 \%$ do grupo com presença de cupim; 88,24\% com presença de plantas invasora em até $15 \%$ da área do pasto; e 94,12\% com altura do capim acima de $12 \mathrm{~cm}$. Esse é o grupo de fazenda com condições de pastagens mais críticas, conforme indicativo de degradação de solo.

O segundo grupo está composto por 13 fazendas com predomínio de ausência de solo exposto em $76,92 \%$ delas e ausência de processo erosivo (84,62\%), portanto menor ocorrência de degradação de solo. Porém, as condições de manutenção e manejo das pastagens e animal estão conduzindo à degradação biológica representada pela presença de cupim em todas essas fazendas, sendo que $53,85 \%$ dessas propriedades tem presença de plantas invasoras em mais de $15 \%$ da área de pastagem. A altura do capim está abaixo de 12 cm em 61,54\% delas, indicando situação de sobrepastejo, comprometendo a recuperação da planta, e deixando o solo mais suscetível aos processos de erosão causados pela chuva. Esse indicativo preocupa pela baixa capacidade de suporte da pastagem, por consequência baixa eficiência produtiva, econômica e ambiental, comprometendo a sustentabilidade da produção em médio e longo prazo, por perda de solo.

O terceiro grupo constituído por 23 fazenda apresenta condição regular de manejos (planta e animal) um pouco melhor que o anterior. $\mathrm{O}$ grupo se caracteriza pela ausência (100\%) de solo exposto e baixa presença de processo erosivo (4,35\%), no entanto $100 \%$ das fazendas têm presenças de cupim e de invasora em até $15 \%$ da área de pastagem. Nesse grupo a pastagem apresenta altura do capim acima de $12 \mathrm{~cm}$, portanto, com melhor massa foliar, por consequência melhor capacidade de suporte animal e proteção do solo.

O quarto grupo de fazendas, menor de todos com $11,67 \%$ do total da amostra, apresenta os menores indicativos de degradação biológica e de solos. Ocorreu ausência total de solo exposto e processo erosivo, indicando que não há degradação de solo nessas fazendas. As menores ocorrências de cupim $(28,57 \%)$ e de invasoras $(57,14 \%)$ em até $15 \%$ da área de pastagem e o predomínio (100\%) da altura do capim acima de $12 \mathrm{~cm}$ sugerem o melhor manejo (planta/animal) do total da pesquisa.

O modelo de regressão beta foi aplicado com a finalidade de obter uma expressão analítica para os índices de degradação de solos e 
degradação biológica. A estimativa do índice de degradação de solo foi constituída pelas variáveis que apresentaram parâmetros estatisticamente significantes, ao nível de significância de 5\%, apresentando proporção de variância explicada pelo modelo de $\mathrm{R}^{2}$ ajustado $=$ $97,43 \%$. Enquanto que as estimativas dos parâmetros da regressão beta para o índice de degradação biológica são estatisticamente significantes, ao nível de significância de $1 \%$, com proporção da variância explicada pelo modelo de $\mathrm{R}^{2}$ ajustado $=96,06 \%$. Utilizou-se das duas componentes estimadas pelos modelos de regressão beta para constituir um único índice de degradação de pastagem, que de acordo com essa amostra gerou cinco categorias, por meio de agrupamentos dos estabelecimentos agropecuários (Figura 5).

Figura 5 - Dispersão dos índices de degradação de solos (Inds) e degradação biológica (Indb), estimadas pela regressão beta, BHRV GO, 2016.

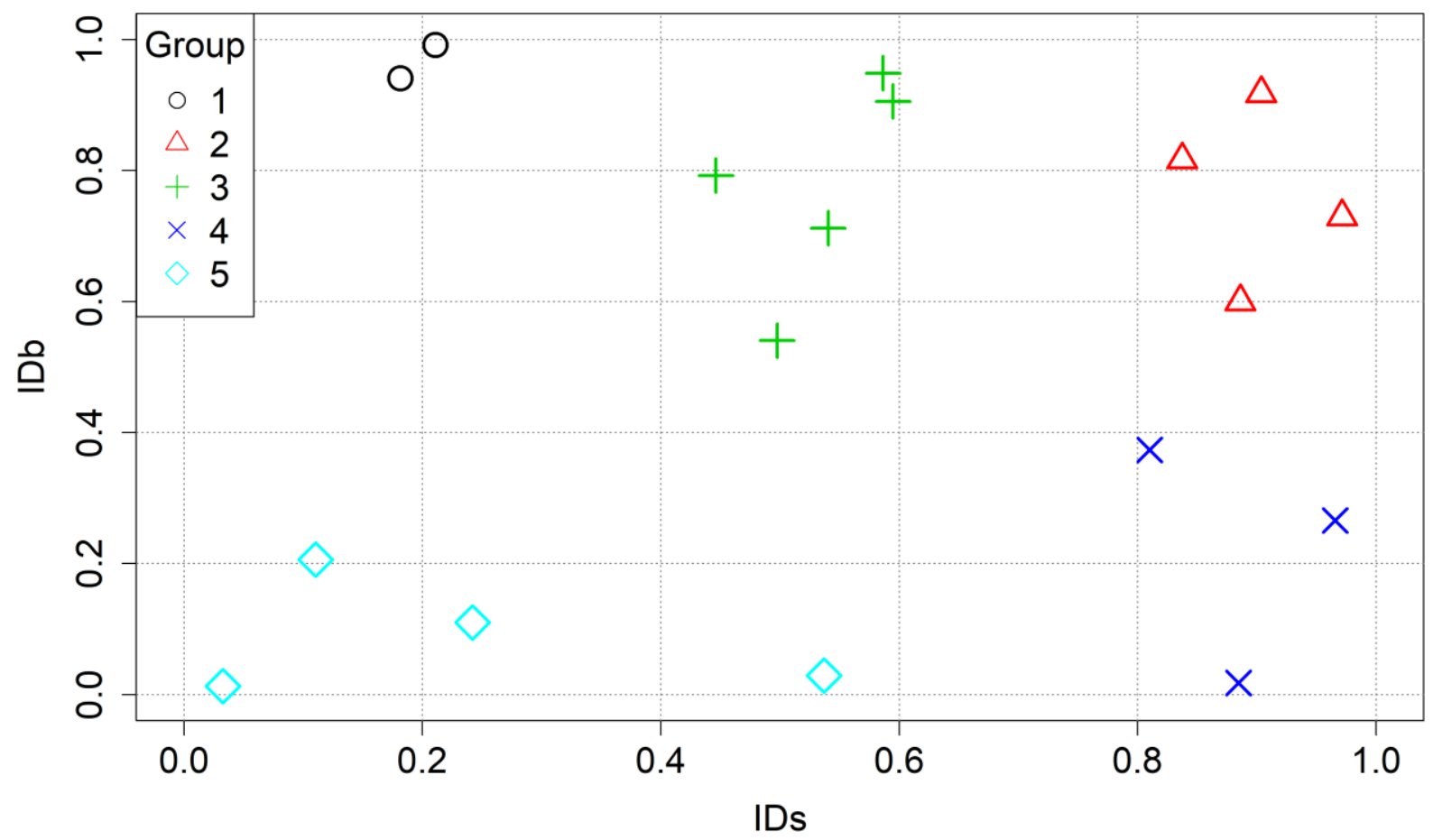

Org:. pelos autores, 2017.

A visualização espacial individual do índice de IDS (Figura 5) possibilitou avaliar as 19 unidades que apresentam alto índice de degradação, sugerindo perda de solo, por solo exposto e processo de erosão hídrica laminar nos pastos. As propriedades com os IDS mais altos estão predominantemente (94,7\%) concentradas na alta e média bacia, onde ocorre com mais frequência relevo ondulado e fortemente ondulado. Quanto à degradação de pastagens, pela dimensão biológica, observa-se que as nove propriedades com mais alto índice estão concentradas $(77,8 \%)$ na baixa e média bacia, enquanto com mais baixo índice concentram-se na alta bacia.

Foram relacionados o IDP (Figura 6 A) e o
IT (Figura 6 B), espacialmente. Ao analisar as 05 categorias resultantes do diagnóstico das condições de degradação de pastagens, representada por IDS e IDB (Figura 6 A), verifica-se que das oito propriedades com os mais baixos IDP (Categoria 1), sete estão concentradas na Alta bacia. Enquanto as 28 propriedades com nível médio de degradação de pastagens (Categoria 3) estão distribuídas em todos os compartimentos. E entre os 5 estabelecimentos com os piores IDP (Categoria 5) três estão concentrados na Média bacia. $\mathrm{Na}$ Baixa bacia, em relação às 12 propriedades pesquisadas predominou (83,3\%) o nível médio de degradação de pastagens. 
Figura 6 - Distribuição espacial da amostra quanto aos indicadores de degradação do solo e biológica (A) e nível tecnológico (B), BHRV GO - Brasil, 2016.

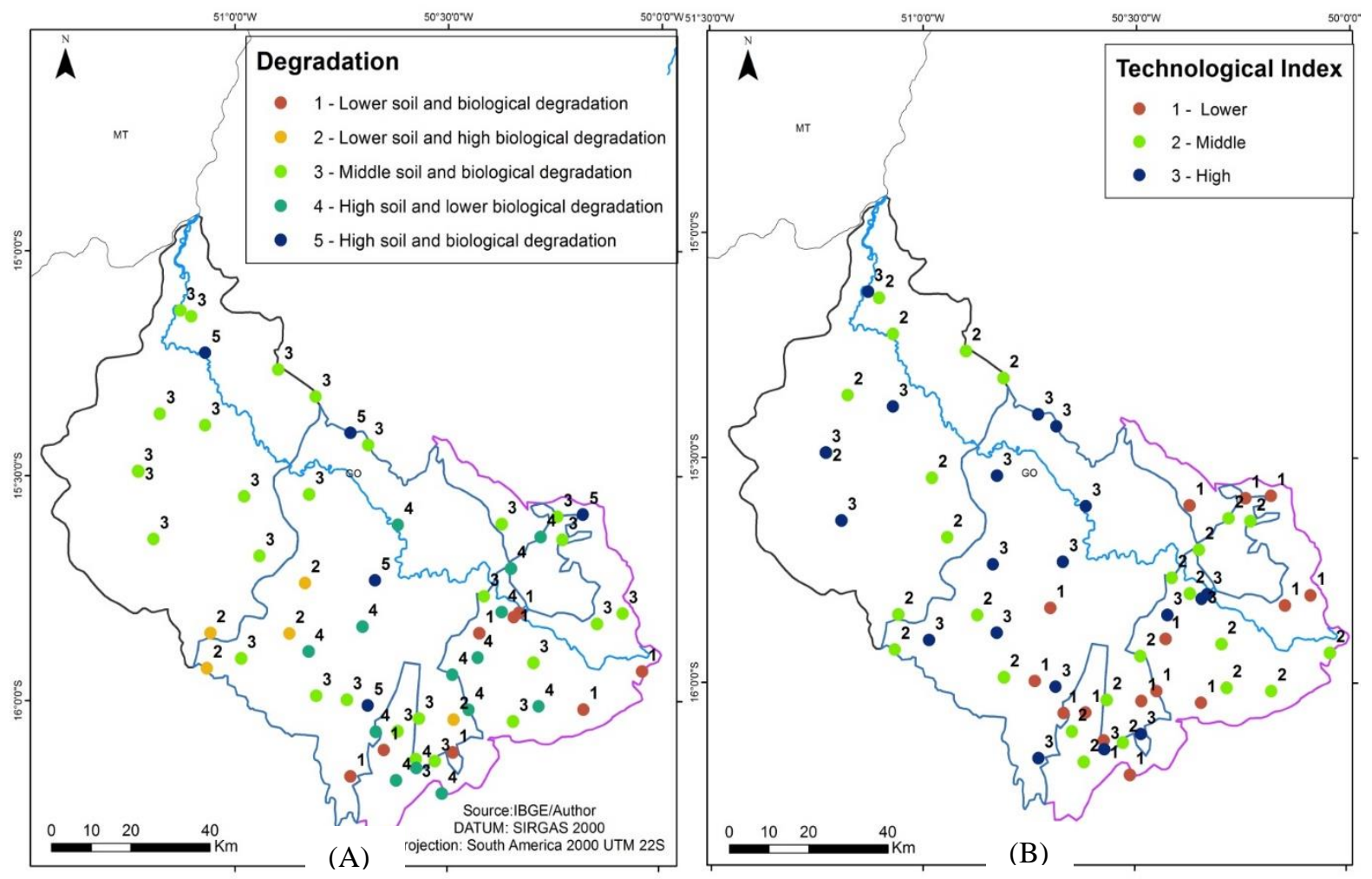

Fonte: IBGE (2017). Oliveira et al. (2018). Org. pelos autores, 2017.

Ao observar a totalidade da bacia, chama a atenção que $46,7 \%$ do total da amostra apresentam nível médio de degradação de pastagens (Categoria 3), e 23,3\% com alto nível de degradação de solos e de baixo nível de degradação biológica (Categoria 4). Enquanto $31,7 \%$ das propriedades apresentam alto nível tecnológico, com maior concentração $(52,6 \%)$ na média bacia. Conforme amostra, na Baixa bacia não ocorreu presença de propriedade com baixo índice tecnológico (Figura 12 B). E a Alta bacia apresenta a maior variabilidade de degradação de pastagens e de nível tecnológico, com maior presença das propriedades que trabalham com baixo padrão de tecnificação. Ressalta-se que nesse compartimento estão concentrados os assentamentos rurais realizados pelo governo, em relação à BHRV.

O índice Global Moran para degradação de pastagens foi de -0,01338 ( $p$-valor $=0,9507, \alpha=$ $5 \%$ ), logo a hipótese nula de ausência de autocorrelação espacial, também, não pode ser rejeitada. Portanto, não há evidência estatística de dependência entre os estabelecimentos agropecuários da região, sugerindo que nível de degradação, assim como nível tecnológico (OLIVEIRA et al., 2018) não está relacionado com a proximidade espacial das propriedades.

\section{Testes das hipóteses}

Para testar as hipóteses foram utilizados os IT e o IDP. Os resultados dos testes aplicados (Tabela 2) revelam os resultados dos testes de correlação entre esses índices e as variáveis.

Em conformidade com os testes aplicados não houve evidencia estatística de associação entre nível tecnológico e grau de escolaridade, sugerindo que a tecnificação praticada na região não depende do grau de educação do gestor, e sim de outras formas de obtenção de conhecimento de técnicas e manejos de produção, inclusive pela assistência técnica. $\mathrm{O}$ uso de assistência técnica pelas propriedades de alto, médio e baixo nível é de 78,9\%, 26,7\% e $13,3 \%$ respectivamente, evidenciando que o produtor com maior nível tecnológico encontra apoio técnico para gestão da atividade. Predominam para todos os níveis tecnológicos gestores com apenas o ensino fundamental. Mesmo com alta escolaridade do gestor, foi observada propriedade com baixo nível tecnológico, quando a propriedade era destinada para lazer. 
Tabela 2. Resultados dos testes estatísticos das hipóteses, BHRV GO, 2016.

\begin{tabular}{|c|c|c|c|c|c|c|c|c|}
\hline \multirow{2}{*}{ Ord } & \multirow{2}{*}{ Hipóteses testadas } & \multirow{2}{*}{$\begin{array}{l}\text { Teste } \\
\text { Exato } \\
\text { Fisher } \\
p \text {-value }\end{array}$} & \multicolumn{2}{|c|}{$\begin{array}{l}\text { Spearman } \\
\text { (g.l:58) }\end{array}$} & \multicolumn{2}{|l|}{$\begin{array}{l}\text { Kendall } \\
\text { (g.l:58) }\end{array}$} & \multicolumn{2}{|c|}{ Kendall $\left(\tau_{c}\right)(g .1: 58)$} \\
\hline & & & $\rho_{s}$ & $\begin{array}{l}p- \\
\text { value }\end{array}$ & $\tau-b$ & $\begin{array}{l}p- \\
\text { value }\end{array}$ & $\tau-c$ & $p$-value \\
\hline 1 & $\begin{array}{l}\text { Ho: IT e grau de educação } \\
\text { do gestor não estão } \\
\text { associados. }\end{array}$ & 0.8319 & 0.1791 & 0.1705 & $\mathrm{Na}$ & na & 0.1575 & 0.1742 \\
\hline 2 & $\begin{array}{l}\mathrm{H}_{0} \text { : IT e nível de renda do } \\
\text { gestor não estão } \\
\text { associados. }\end{array}$ & 0.2889 & 0.2525 & $\begin{array}{l}0.0519 \\
(*)\end{array}$ & $\mathrm{Na}$ & na & 0.2067 & $\begin{array}{l}0.0314 \\
(* *)\end{array}$ \\
\hline 3 & $\begin{array}{l}\mathrm{H}_{0} \text { : IT e crédito rural não } \\
\text { estão associados. }\end{array}$ & 0.3568 & -0.1529 & 0.2428 & $\mathrm{Na}$ & na & -0.1533 & 0.2472 \\
\hline 4 & $\begin{array}{l}\mathrm{H}_{0} \text { : IT e relevo percebido } \\
\text { pelos respondentes não } \\
\text { estão associados. }\end{array}$ & -0.2976 & -0.2195 & $\begin{array}{l}0.0921 \\
(*)\end{array}$ & -0.2021 & $\begin{array}{l}0.0860 \\
(*)\end{array}$ & na & $\mathrm{Na}$ \\
\hline 5 & $\begin{array}{l}\mathrm{H}_{0}: \text { IDP e IT não estão } \\
\text { correlacionados. }\end{array}$ & 0.4000 & -0.1390 & 0.2887 & $\mathrm{Na}$ & na & -0.1250 & 0.2969 \\
\hline 6 & $\begin{array}{l}\mathrm{H}_{0}: \text { IT e tamanho da } \\
\text { propriedade não estão } \\
\text { associados. }\end{array}$ & $\begin{array}{l}0.0019 \\
(* * *)\end{array}$ & 0.4491 & $\begin{array}{l}0.0003 \\
(* * *)\end{array}$ & 0.3966 & $\begin{array}{l}0.0000 \\
(* * *)\end{array}$ & na & $\mathrm{Na}$ \\
\hline 7 & $\begin{array}{l}\mathrm{H}_{0}: \text { IDP e tamanho da } \\
\text { propriedade não estão } \\
\text { associados. }\end{array}$ & 0.5469 & -0.1026 & 0.4342 & $\mathrm{Na}$ & na & -0.0900 & 0.4266 \\
\hline 8 & $\begin{array}{l}\text { H0: Taxa de lotação e IT } \\
\text { não estão associados. }\end{array}$ & na & 0,0875 & 0,5052 & $\mathrm{Na}$ & na & na & na \\
\hline
\end{tabular}

A hipótese de não associação $\left(\mathrm{H}_{0}\right)$ entre nível tecnológico e renda do gestor foi rejeitada, conforme resultados dos testes de Spearman $\left(\rho_{s}\right.$ $(58)=0,2525, p<0,10)$ e Kendall $\left(\tau_{c}(58)=\right.$ 0,2067, $p<0,10)$, indicando relação de dependência entre elas. Ao modelar as variáveis: índice tecnológico e renda pela regressão beta, com a finalidade de melhor explorar a relação de dependência entre elas verificou-se que os níveis de renda com 5 a 10 salários mínimos (Renda 3); e acima de 10 salários (Renda 4) se mostraram significativas $(p<0,10)$ para estimar nível tecnológico, com contribuições positivas e crescentes. Entre as 19 fazendas com maior índice tecnológico, 10 proprietários $(52,6 \%)$ administram diretamente o estabelecimento agropecuário com área mediana de 469,48 hectares, podendo ou não ter apoio de empregado para a função de gerente. E entre esses dez proprietários respondentes, observou-se que 50\% residem em área urbana e deslocam para a fazenda; $60 \%$ vivem exclusivamente com a renda agropecuária.

No grupo de maior nível tecnológico, 73,7\% estão vinculados ao sindicato rural. Entre os proprietários, $60 \%$ dependem exclusivamente da renda agropecuária. Em média menos de $40 \%$ dos gestores contribuem para a previdência oficial, revelando que a maioria está à margem da proteção previdenciária. Ressalta-se que $13,3 \%$ de todos os 60 entrevistados já vivem com um benefício concedido pela previdência, suplementando a renda da atividade agropecuária.

De acordo com a amostra, a principal forma de acesso a terra na BHRV é por meio de herança (53,3\%), aquisição $\quad(40,0 \%)$ e assentamento (6,7\%). O grupo de estabelecimentos com alto índice tecnológico, diferente dos demais, apresenta a aquisição $(52,6 \%)$ como principal forma de acesso a terra e com maior proporção de propriedades com administração realizada por empregados $(47,4 \%)$, com perfil de produção empresarial.

Para o teste de hipótese IT e uso de crédito rural não foi observada evidência estatística de dependência. $\mathrm{O}$ crédito rural, obtido por meio de programas governamentais, amplia a capacidade financeira de custeio e de investimento, potencializando melhorias tecnológicas para os sistemas de produção. E o uso dele, em relação ao total da amostra, foi menor $(8,3 \%)$ para o grupo com IT mais elevado 
e igual para os demais grupos (11,7\%). A baixa utilização de crédito rural pelos estabelecimentos com maior IT sugere que estão usando recursos próprios para custeio e investimentos na produção de bovinos.

A hipótese nula de independência entre nível tecnológico e relevo, percebido pelo gestor, pode ser rejeitada, conforme testes de Spearman $\left(\rho_{s}(58)=-0,2195, p<0,10\right)$ e Kendall $\left(\tau_{b}(58)=-0,2021, p<0,10\right)$, evidenciado a relação de dependência entre as variáveis, ratificando as observações constantes da literatura nacional e internacional sobre relevo plano e suave ondulado são mais favoráveis à aplicação de tecnologias com melhor resposta de produtividade, tanto pelos aspectos naturais de solos, declividade, suscetibilidades à erosão, quanto ao uso de máquinas (GOENER et al., 2007; GRIFFITH, 2016). A associação negativa entre as variáveis indica que em relevos planos a suavizados (Baixa e Média bacia) concentram propriedades com maior nível tecnológico, enquanto que relevos mais fortemente ondulados estão associados com propriedades com baixo padrão tecnológico (Alta bacia).

A relação de dependência entre IT e relevo, analisada pela regressão beta evidencia revelaque apenas a variável relevo3 (relevo fortemente ondulado), presente principalmente no compartimento da alta bacia, apresentou-se significativa $(p<0,05)$. A contribuição de relevo fortemente ondulado para o modelo é negativa e decrescente, indicando que quanto mais fortemente movimentado o relevo menor será o IT estimado.

A hipótese nula de independência entre IT e IDP não pode ser rejeitada, para nenhum nível de significância considerado, sugerindo independência entre elas, com possível ocorrência de degradação de solos e/ou de pastagens em propriedades com qualquer um dos três padrões tecnológicos estabelecidos.

O nível tecnológico está associado com o tamanho de propriedade, conforme resultados dos três testes estatísticos (Fisher, $p<0,01$ ); Spearman $\left(\rho_{s}(58)=0,4491, p<0,01\right)$ e Kendall $(\tau b(58)=0,3966, p<0,01)$, sendo a hipótese nula rejeitada com menor nível de significância (1\%). Grandes propriedades (acima de 675 hectares) estão diretamente relacionadas com o maior IT, enquanto que as menores propriedades (abaixo de 180 ha) estão associadas com o padrão mais baixo de tecnologia. Essa relação restrita ao nível tecnológico dos sistemas de produção de bovinos da BHRV está em consonância com o processo de produção empresarial da pecuária brasileira, principalmente de gado de corte em maior escala, que demanda maior área de pastagens, conforme sistema predominante de produção a pasto (ARMENTERAS et al., 2013; GIBBS et al., 2010).

Relação mais minuciosa entre IT e tamanho da propriedade mostra que "propriedade média" (tamanho2) com a área total na faixa de $180 \leq$ área $<675$ hectares, e "propriedade grande" (tamanho3), acima de 675 hectares foram significativas $(p<0,01)$ e $(p<0,001)$, respectivamente. As contribuições das duas variáveis preditoras contribuem de forma positiva e crescente com a variável resposta, indicando que quanto maior a área maior será o efeito do tamanho da fazenda sobre o índice tecnológico. Conforme dados coletados, ao analisar as frequências do tamanho de propriedades verifica-se a concentração em grandes $(42,1 \%)$ e médias $(31,6 \%)$, com alto padrão tecnológico. Enquanto com baixo nível de tecnificação predominam as pequenas propriedades $(86,7 \%)$.

Não foi observada evidência estatística para associação entre degradação de pastagens e tamanho de propriedade, conforme testes aplicados, sugerindo que os pastos podem apresentar nível de degradação independente da classificação de propriedade em pequena, média ou grande.

Também, não houve evidência estatística quanto à associação entre taxa de lotação e IT, sugerindo que alta taxa de lotação pode ser observada nos três níveis de tecnificação. Ocorrência de taxa de lotação elevada em sistemas com baixo nível tecnológico conduz à degradação de pastagens de forma mais acelerada (pelo sobrepastejo, pisoteio intensivo, entre outros problemas ambientais), resultando em baixa eficiência produtiva, econômica e maus tratos com os animais (OLIVEIRA et al., 2018).

\section{CONCLUSÕES}

A dimensão socioeconômica em que o produtor está inserido e sua relação de dependência com o padrão de tecnificação foi analisada considerando: educação, renda e uso de crédito rural. Verificou-se (considerando o teste exato de Fisher e os testes de correlação de Spearman e Kendall $\tau_{c}$ ) que não há evidencia estatística de associação entre as variáveis: grau de escolaridade e nível tecnológico. No entanto, predomina a experiência dos gestores, com produção de bovinos, por mais de oito anos. 
Entretanto o nível de renda dos gestores e o IT apresentou relação de dependência (considerando testes de correlação de Spearman e Kendall $\tau_{c}$, e análise de regressão beta), sugerindo que propriedades com maior nível tecnológico remuneram melhor seu gestor.

As condições de relevo percebidas pelo gestor e nível tecnológico estão associados negativamente, conforme evidenciado pelo teste de Spearman e Kendall $\tau_{b}$.

IT e taxa de lotação não são dependentes, sugerindo a possibilidade de ocorrência de alta taxa de lotação em propriedades com baixo índice tecnológico, resultando em parâmetros zootécnicos baixos, degradação de pastagens, comprometendo o bem-estar animal. Assim, não é recomendável o uso isolado dessa variável para análise do nível tecnológico dos estabelecimentos agropecuários.

IT está associado com o tamanho da propriedade, conforme resultados dos testes de Fisher, Spearman e Kendall $\tau_{b}$. verificou-se o predomínio de médias e grandes propriedades na baixa e média bacia em relação à alta bacia. A análise mais detalhada de dependência entre as duas variáveis (pela regressão beta) indica que os dois maiores tamanhos de propriedade (médio e grande) contribuem de forma positiva e crescente para o IT. Esse resultado está coerente com a produção em grande escala de gado de corte, baseado em sistema de pastejo, por demandar maior área.

A construção do IDP revelou dois agrupamentos (degradação de solo e biológica) relevantes para a mensuração do mesmo, que analisados em conjunto mostram que $78,3 \%$ da amostra apresenta indicativo de médio a alto nível de degradação.

Não houve evidencia estatística de associação entre IT e IDP (considerando Fisher, Spearman e Kendall $\tau_{c}$ ). Esse resultado sugere que degradação de pastagens está ocorrendo em estabelecimento independente do nível de tecnificação.

\section{AGRADECIMENTOS}

Agradecemos à Fundação de Pesquisa de Goiás (FAPEG) pelo financiamento do programa de integração (PRONEX / FAPEG / CNPq Programa de Apoio aos Núcleos de Excelência), do qual está pesquisa faz parte; à Coordenação de Aperfeiçoamento de Pessoal de Ensino Superior (CAPES) e FAPEG pelas bolsas de estudo; e ao apoio da Fundação Gordon e Betty Moore.

\section{REFERÊNCIAS}

ARMENTERAS, D., et al. Landscape Dynamics in Northwestern Amazonia: An Assessment of Pastures, Fire and Illicit Crops as Drivers of Tropical Deforestation. PLoS ONE, v.8, January, 2013. https://doi.org/10.1371/journal.pone.0054310

BOWMAN, M., et al. Persistence of Cattle Ranching in the Brazilian Amazon: A Spatial Analysis of the Rationale for Beef Production. Land Use Policy, v. 29, p.558-568, 2012. https://doi.org/10.1016/j.landusepol.2011.09.0 09

CARO, D., et al. Greenhouse Gas Emissions Due to Meat Production in the Last Fifty Years. In: Ahmed M., Stockle C. (eds) Quantification of Climate Variability, Adaptation and Mitigation for Agricultural Sustainability. Springer, Cham, 2017. https://doi.org/10.1007/978-3-319-32059-5_2

DIAS, L.C.P., et al. Patterns of land use, extensification, and intensification of Brazilian agriculture. Global change biology, v.22, n. 8, p.2887-2903, 2016. https://doi.org/10.1111/gcb.13314

GIBBS, H. K., et al. Tropical forests were the primary sources of new agricultural land in the 1980s and 1990s. Proceedings of the National Academy of Sciences, v. 107, n.38, p.16732-16737, 2010. https://doi.org/10.1073/pnas.0910275107 GOENER, A., GLOAGUEN, R., MAKESCHIN, F. Monitoring of the Ecuadorian mountain rainforest with remote sensing. Journal of Applied Remote Sensing, v.1, n. 1, p.013527-013527-12, 2007. https://doi.org/10.1117/1.2784111

GREENACRE, M.. Correspondence Analysis in Practice. Barcelona, Chapman and Hall/CRC, 2007. https://doi.org/10.1201/9781420011234

GRIFFITH, D. C. Migration, Labor Scarcity, and Deforestation in Honduran Cattle Country. Journal of Ecological Anthropology, v. 18, n. 1, p.3, 2016. https://doi.org/10.5038/2162-4593.18.1.3

GUEDES, T. A., et al. Seleção de variáveis categóricas, utilizando análise de correspondência e análise procrustes. Acta Scientiarum. Technology, v.21, p.861-868, 2008.

https://doi.org/10.4025/actascitechnol.v21i0.30 84

HAIR JR., J.F., et al. Análise multivariada de dados. Trad. Adonai Schlup Sant'Anna e 
Anselmo Chaves Neto. 5 ed. Porto Alegre: Bookman, 2009.

IBGE - Instituto Brasileiro de Geografia e Estatística. Base Cartográfica Contínua do Brasil ao milionésimo. Ver 3. Rio de Janeiro. 2017. Available in: < https://bit.ly/2EROYLT>. Acessed: february, 12, 2018.

Economia. Censo agropecuário, 2006. Available in: <https://bit.ly/3d0SazL>. Acessed: july, 05, 2017.

KLINK, C.A., MACHADO, R.B. A conservação do Cerrado brasileiro. Megadiversidade, v.1, n. 01, p.147-155, 2005. Available in: < https://bit.ly/3g9rUFa>. Acessed: August 26, 2018.

LIMA, S. S., et al. Relação entre a presença de cupinzeiros e a degradação de pastagens. Pesquisa Agropecuária Brasileira, v.46, n.12, p.1699-1706, 2012. https://doi.org/10.1590/S0100204X2011001200016

LIMA, V. M. A., CALDARELLI, C. E., CAMARA, M. R. G. Análise do desenvolvimento municipal paranaense: uma abordagem espacial para a década de 2000.

Economia e Desenvolvimento, v. 26, n. 1, p. 1-19, 2014. https://doi.org/10.5902/1414650911030

MACHADO, L.E.G., LIMA, C.V.L. Compartimentação geomorfológica da bacia hidrográfica do Rio Vermelho (GO), utilizando imagens ASTER. In: Simpósio Brasileiro de Sensoriamento Remoto - SBSR, Curitiba. Anais... São Paulo, Instituto Nacional de Pesquisa Espacial, p.8231, 2011. Available in: $<$ https://bit.ly/2NDPOwj>. Acessed: August 26, 2018.

MARGULIS, S. Causas do Desmatamento da Amazônia Brasileira. Banco Mundial Brasília DF, 2003. Available in: < https://bit.ly/3d0SSNr >. Acessed: August 26, 2018.

MCMANUS, C., et al. Dynamics of Cattle Production in Brazil. Plos One, January 27, 2016.

https://doi.org/10.1371/journal.pone.0147138

OECD-FAO - Organization for Economic Cooperation and Development, Food and Agriculture Organization of the United Nations, 2015. OECD-FAO Agricultural Outlook 2015 - Chapter 2. Paris. http://dx.doi.org/10.1787/agr_outlook-2015-4-

pt

OLIVEIRA, E. R., et al. Development of a technological index for the assessment of the beef production systems of the Vermelho River Basin in Goiás, Brazil. Revista
Pesquisa Operacional, v.38, n.1, p.117-134, 2018. https://doi.org/10.1590/01017438.2018.038.01.0117

PAGÈS, J. Multiple Factor Analysis by example using $\mathbf{R}$. Chapman and Hall, CRC Press, 2014.

PERON, A.J.; EVANGELISTA, A.R. Degradação de pastagens em regiões de cerrado. [Degradation of pasture in the Brazilian Cerrado]. Revista Ciência e Agrotecnologia, Lavras, MG, v. 28, n. 30, p.655-661, 2004 . https://doi.org/10.1590/S141370542004000300023

PINHEIRO, T. F. et al. Forest degradation associated with logging frontier expansion in the Amazon: the BR-163 region in Southwestern Pará, Brazil. Earth Interactions, v.20, n. 17, p.1-26, 2016. https://oi.org/10.1175/EI-D-15-0016.1

ROSA, A. N. et al. Peso adulto de matrizes em rebanhos de seleção da raça Nelore no Brasil. Revista Brasileira de Zootecnia, v.30, n.30, p. 1027-1036, 2001. https://doi.org/10.1016/j.rsase.2020.100288

SHIRVANI, Z., et al. Analyzing spatial and statistical dependencies of deforestation affected by residential growth: Gorganrood basin, Northeast Iran. Land Degradation \& Development, 18 april, 2017. https://doi.org/10.1002/ldr.2744

WARD JR., J.H. Hierarchical grouping to optimize an objective function. Journal of the American statistical association, v.58, n.301, p.236-244, 1963. https://doi.org/10.1080/01621459.1963.105008 45 